\title{
An AIAD-Based Adaptive Routing Protocol in Ad-Hoc Wireless Networks
}

\author{
Youn-Sik Hong ${ }^{1}$ and Ki-Young Lee ${ }^{2}$ \\ ${ }^{1}$ Department of Computer Science and Eng. \\ ${ }^{2}$ Department of Information and Telecommunication Eng., \\ University of Incheon \\ 177 Dowha-dong Nam-gu 402-749 Incheon, Korea \\ \{yshong, kylee\} aincheon.ac.kr
}

\begin{abstract}
AODV routing protocol is intended for use by mobile nodes in adhoc wireless networks. Even though it performs well in static and low-mobility environments, the performance degrades rapidly with increasing mobility. Our primary concern is to enhance the performance of AODV by reducing the volume of the control packets like RREQ and RREP during the route discovery process effectively due to the node mobility. The propagation delays of the all possible links vary according to changes in its topology. Carefully adjusting the values of these network parameters can reduce the occurrences of the control packets. Thus, we propose a novel method of smoothly adjusting them based on AIAD (additive increase additive decrease) under a consideration of current network status. We have tested our proposed method with both the conventional AODV and the method using timestamp based on the three performance metrics; i.e., node mobility, node velocity, and node density, to compare their performances.
\end{abstract}

Keywords: Ad-hoc Network, AODV, Expanding Ring Search, Node Mobility, Node Traversal Time.

\section{Introduction}

Ad-hoc wireless network architecture is a self-organizing and distributed controlled network formed by a set of stations (called nodes) that can freely and dynamically self-configure and organize themselves to set up a temporary wireless network [1]. In the ad-hoc network configuration, a node acts as a mobile terminal as well as a router to forward messages to neighboring nodes if possible. The network topology in an ad hoc network is highly dynamic due to the movement of nodes; hence an on-going session suffers frequent path breaks. A disruption may be occurred either due to the movement of the intermediate nodes in the path or due to the movement of end nodes. Routing protocols for ad-hoc networks must be able to perform efficient and effective mobility management.

Existing ad-hoc routing protocols may generally be categorized as table-driven and source-initiated demand driven [2]. In table-driven routing protocols, every node maintains the network topology information in the form of routing tables by 
periodically exchanging routing information. These protocols suffer from excessive control overhead that is proportional to the number of nodes in the network and therefore is not scalable in ad-hoc wireless networks, which have limited bandwidth and whose topologies are highly dynamic.

Unlike the table-driven routing protocols, on-demand routing protocols execute the path-finding process and exchange routing information only when a path is required by a source node to communicate with a destination. One of the on-demand routing protocols is AODV (Ad-hoc On-demand Distance Vector) [5]. In AODV, a source node floods the Route Request (RREQ) packet in the network when a route is not available for the desired destination. When an intermediate node receives a RREQ, it either forwards it or prepares a Route Reply (RREP) if it has a valid route to the destination. All intermediate nodes having valid routes to the destination, or the destination node itself, are allowed to send RREP packets to the source. AODV reduces the need for the system wide broadcasts by localizing the propagation of changes in the network. Even though it performs well in static and low-mobility environments, the performance degrades rapidly with increasing mobility.

As shown in Fig 1, data packet transmission is slightly affected by the node mobility. However, as the node mobility increases the control packets like RREQs and RREPs increase rapidly. Then it causes route discovery latency to increase. Besides, the amount of energy consumption increases corresponding to the increased number of control packets. To the end, the overall performance should be degraded with high node mobility. Our primary concern is to enhance the performance of AODV by reducing the volume of these control packets effectively due to the node mobility.

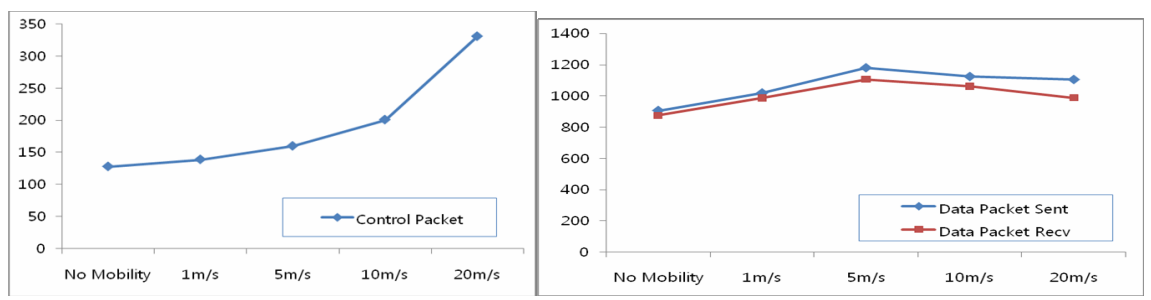

Fig. 1. The number of control packets (left) and the number of data packets (right) with node mobility

The rest of this paper is organized as follows. In section 2, we describe the expanding ring search algorithm and related works. In section 3, we present a proposed approach based on AIAD method. In section 4, a series of experimental results will be given and finally we conclude our paper in section 5 .

\section{Expanding Ring Search and Related Works}

\subsection{Expanding Ring Search}

Consider the example depicted in Fig 2. In this figure, the source node 1 initiates a path-finding process by originating a RREQ to be flooded in the network for the 

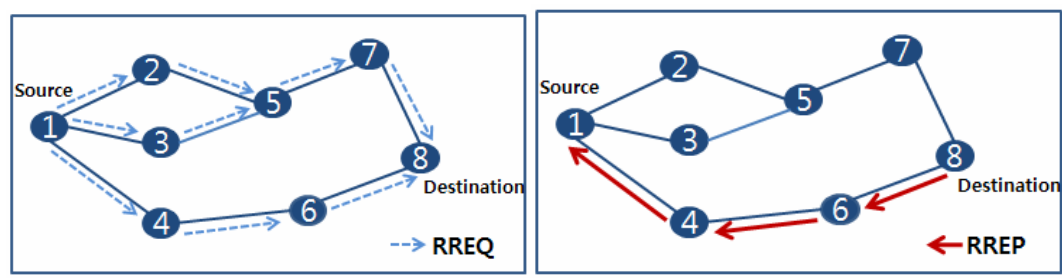

Fig. 2. AODV route discovery; propagation of the RREQ packets (left) and a path of the RREP packet to the source (right)

destination node 8 . When the intermediate nodes 2,3 , and 4 receive the RREQ packet, they check their routes to the destination. In case a route to the destination is not available, they further forward it to their neighbors. If the RREQ reaches the node 8 through path 1-4-6-8 or any other alternate route, it also sends a RREP to the source.

To prevent unnecessary network-wide dissemination of RREQs, the source node should use an expanding ring search (ERS) technique [6]. Centered on the source node, ERS successively searches larger areas until a node with the information being searched is located. In the ERS, the source node initially uses a TTL (time to live) = TTL_START in the RREQ packet header and sets the timeout for receiving a RREP to RING_TRAVERSAL_TIME milliseconds [5]. If the RREQ times out without a corresponding RREP, the source node broadcasts the RREQ again with the TTL incremented by TTL_INCREMENT. This continues until the TTL set in the RREQ reaches TTL_THRESHOLD, beyond which a TTL = NET_DIAMETER is used for each attempt. Beyond this, the RREQ is not forwarded any further. Each time, the timeout for receiving a RREP is RING_TRAVERSAL_TIME. Table 1 gives default values for some important parameters associated with ERS techniques used in the AODV routing protocol.

Table 1. Default values for AODV parameters

\begin{tabular}{cc}
\hline Parameter name & value \\
\hline NET_DIAMETER & 35 \\
NODE_TRAVERSAL_TIME & $40 \mathrm{~ms}$ \\
NET_TRAVERSAL_TIME & $2 \times$ NODE_TRAVERSAL_TIME $\times$ NET_DIAMETER \\
RING_TRAVERSAL_TIME & $2 \times$ NODE_TRAVERSAL_TIME $\times$ TTL_VALUE \\
TTL_START & 1 \\
TTL_INCREMENT & 2 \\
TTL_THRESHOLD & 7 \\
\hline
\end{tabular}

NODE_TRAVERSAL_TIME (NTT) is a conservative estimate of the average one hop traversal time for packets and should include queuing delays, interrupt processing times and transfer times [5]. Typically NTT is set to a fixed value of 40ms. In that case, it does not reflect a full dynamic topology with respect to node mobility in adhoc wireless networks. For example, node $i$ moves to the right in some distance from its neighbors as shown in Fig 3, while it still resides in a transmission range of them. 
It causes a longer response time for the query from its neighbors. In TTL-based ERS, if the node cannot reply within a specified time-out period, the source node reinitiates a RREQ packet as shown in Fig 3(a). However, by setting the appropriate TTL value in the query as shown in Fig 3(b), the source node can control the search radius. Thus, by setting the appropriate NTT value the unnecessary RREQ packets should be minimized. Minimizing the cost of the initiating and the forwarding RREQ packets is crucial for resource-constraint multi-hop wireless network, which motivates this work.

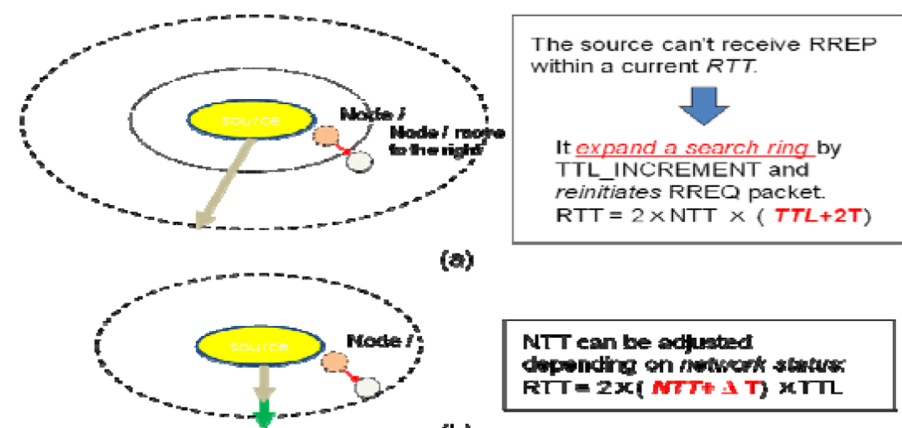

(b)

Fig. 3. An illustration of our proposed approach: (a) TTL-based ERS, (b) NTT-based ERS

\subsection{Related Works}

A number of heuristics for enhancements of the performance of AODV routing protocol has been proposed. Hassan and Jha [7] explored to find an optimum TTL threshold $L$ that would minimize the expected bandwidth cost of ERS. They give the following experimental results; search threshold L of 3 is optimum for three categories of networks, that is, large networks with small radius, medium networks with large radius and medium networks with small radius. In the AODV, TTL_THRESHOLD is typically set to 7 , which gives the search threshold of 3 .

Some of the works tries to identify the inefficient elements of ERS. Lee et al[9] proposed a method of timestamp to compute NTT; every node adds the current time to the message (i.e., RREQ and RREP) before sending it out, and then the neighbor that receives it computes a measured traversal time (called $M$ ) using the timestamp. A smoothed NTT is updated by using the similar equation of the round-trip-time estimator:

$$
\mathrm{NTT}=\alpha \times \mathrm{NTT}+(1-\alpha) \mathrm{M}
$$

where, $\alpha$ is a smoothing factor with a range of 0 and 1. Kim et al [10] presented a technique of estimating NTT that divides a time difference between the source node and the destination node by the number of hops. In addition, Park et al [11] proposed a blocking ERS (B-ERS for short). One of the differences from the TTL-ERS is that the B-ERS does not resume its route search procedure from the source node each time a rebroadcast is required. Tripathi et al [8] proposed four heuristics; one for them is the utilization of route caches at intermediate nodes for route discovery. 
All of the works mentioned above have an emphasis on the adaptive control of AODV routing protocol with respect to node mobility. Since the timestamp based approaches [9] changes NTT too rapidly, the stability of the network becomes relatively low. Besides, with low mobility their performance will be degraded due to increases in the number of RREQ packets initiated by the source node. Even if B-ERS reduces in route discovery latency, it may suffer from dynamic changes in the network topology with high mobility. With the proposed heuristics proposed by Tripathi et al, the complexity of maintenance of multiple paths demands a larger memory and greater processing power for every node.

\section{A Control Packet Minimized AODV Protocol}

Our proposed method relies on packet based propagation of timestamp information to calculate more realistic NTT by considering the network status. While initiating a RREP, the destination node appends the timestamp information in the RREP packet. When the source node receives the RREP packet, it checks the timestamp ST available at the source node and the timestamp DT available at the destination node. Let $M T$ denotes estimated time taken for a packet to be delivered from one node to one-hop neighboring node. Let the number of hops on the route path established between the source node and the destination node be denoted by $\mathrm{H}$. Then the $M T$ is calculated as:

$$
\mathrm{MT}=(\mathrm{ST}-\mathrm{DT}) / \mathrm{H}
$$

A computed estimate $M T$ of one-hop traversal time just gives a reference relative to a previous NTT. A new NTT should be adjusted depending on MT. For example, the path 8-6-4-1 in Fig. 2 consists of three links. Assume that each of them has a propagation delay of $40 \mathrm{~ms}, 30 \mathrm{~ms}$, and $20 \mathrm{~ms}$, respectively. In that case, MT is computed as $30 \mathrm{~ms}$. However, since the longest propagation delay should be considered, a NTT is not changed and still remains $40 \mathrm{~ms}$. If the propagation delays of the all links increase equally in $6 \mathrm{~ms}$ due to node mobility, MT is $36 \mathrm{~ms}$ and then the amount of the difference between NTT and MT becomes smaller. Thus, a new NTT should be increased by a certain amount $\Delta$.

Table 2. The recalculated NTT with respect to the propagation delays of each link (unit: ms)

\begin{tabular}{llllccc}
\hline \multicolumn{2}{c}{ Propagation delay } & \multirow{2}{*}{ MT } & $\begin{array}{c}\text { previous } \\
\text { NTT }\end{array}$ & $\begin{array}{c}\text { NTT - } \\
\text { MT }\end{array}$ & New NTT \\
\hline 40 & 30 & 20 & 30 & 40 & 10 & 40 \\
46 & 36 & 26 & 36 & 40 & 4 & $40+\Delta$ \\
34 & 24 & 14 & 24 & 40 & 16 & $40-\Delta$ \\
\hline
\end{tabular}

On the contrary, if the delays of the all links decrease equally in $6 \mathrm{~ms}$, MT is $24 \mathrm{~ms}$ and then the amount of difference between them becomes greater. So a new NTT should be decreased by $\Delta$. The above explanations are summarized as shown in Table 2 .

Then the following algorithm, called CP_AODV (Control Packet minimized AODV), is used to resolve the number of control packets including RREQ and RREP packets. To minimize the flooding of control packets, a NTT should be adjusted smoothly by AIAD (additive increase additive decrease) technique. 


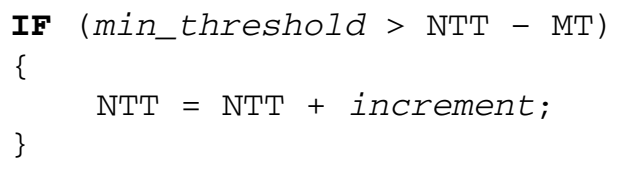

The parameters min_threshold, max_threshold, and increment are adjustable with respect to the network status. Notice that max_threshold $>$ min_threshold. When the amount of the difference between NTT and MT is less than min_threshold, the average propagation delay of the links seems to be increased. So a new NTT should be increased by a specific amount increment. On the contrary, when the amount of the difference between them is greater than max_threshold, the average delay seems to be shorter. Then a new NTT should be decreased by increment. The parameter increment is controlled adaptively depending on the movement of nodes. Our experimental results show that with less than 40ms of NTT the number of retransmission of the RREQ packets increases so rapidly and thus the overall performance is degraded. Thus, the lower bound on NTT is set to $40 \mathrm{~ms}$. While the amount of difference between them is in the range of min_threshold and max_threshold, the node mobility seems to be relatively low. Then the value of a current NTT remains as before.

\section{Performance Evaluations}

For the purpose of the simulation, AODV protocol implemented in QualNet 4.0 simulator [12] has been modified to incorporate the changes which have been proposed in this paper. Data payload is of 512 bytes. All the data packets are CBR (continuous bit rate) packets. Each node moves with randomly chosen maximum speed up to $10 \mathrm{~m} / \mathrm{s}$. The simulation parameters are summarized as shown in Table 3. Notice that the control parameters used in the CP_AODV are set through our simulations as the following: min_threshold $=5$, max_threshold $=10$, increment $=5$.

Table 3. Simulation set-up

\begin{tabular}{cc}
\hline attributes & value \\
\hline space & $1,500 \mathrm{~m} \times 1,500 \mathrm{~m}$ \\
bandwidth & $2 \mathrm{MB}$ \\
Number of nodes & 49 \\
Placement strategy & GRID \\
Transmission range & $250 \mathrm{~m}$ \\
Node velocity & $0 \sim 10 \mathrm{~m} / \mathrm{s}$ \\
run time & $600 \mathrm{~seconds}$ \\
\hline
\end{tabular}


The performance evaluation is based on the comparison of the three different metrics; node mobility, node velocity and node density. In order to get a realistic insight into the effects of the proposed method (CP_AODV), these metrics also have been evaluated for both the legacy AODV [6] and the modified AODV with the timestamp method [9] (T-AODV for short).

\subsection{The Experiments for the Node Mobility}

After reaching the destination the node begins to move again after pause time. In our experiments we varied it between 0 to 600 seconds. The network between the pause times 0 to 150 have a high mobility, whereas the network beyond 450 have a low mobility. The network between the pause times 150 to 450 have a moderate mobility. The pause time implies the level of the mobility in the network.

We classify RREQ messages into two types; a RREQ initiated by the source node and a RREQ forwarded by an intermediate node. The source node re-initiates the RREQ if there is no route reply within a time-out period. Thus, a possible measurement to evaluate the cost of network-wide flooding is the number of the RREQ packets initiated by the source node. RREQ packets are flooded in the network by intermediate nodes forwarding the requests to their neighbors. So, another measurement is the number of RREQ packets forwarded by them.

For the number of RREQ packets initiated by the source node, CP_AODV is remarkably less than AODV as shown in Fig 4. In addition, CP_AODV is better than

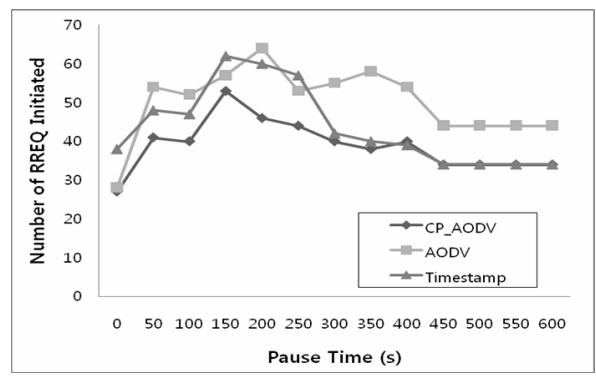

Fig. 4. The number of RREQ packets initiated by a source node

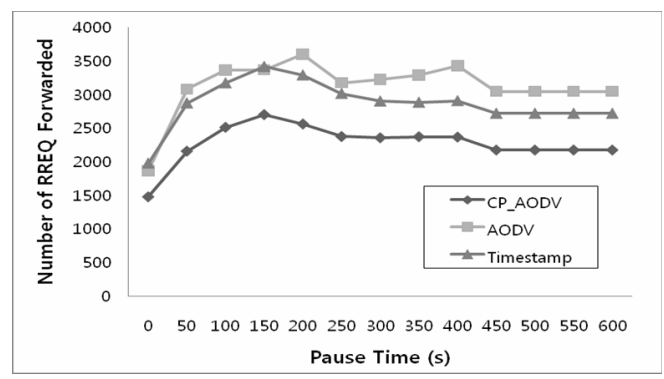

Fig. 5. The number of RREQ forwarded by intermediate nodes 
T-AODV except in low mobility. For the number of RREQ packets forwarded by intermediate nodes, $\mathrm{CP} \_$AODV has significantly lower average packets than the two protocols (Fig 5). Typically, with CP_AODV the number of RREQ packets forwarded is reduced by $28 \%$ and $21 \%$ compared to AODV and T-AODV, respectively.

The decreased number of RREQ packets result in the decreased number of both RREP and RERR. For the number of RREP packets initiated by the destination node, CP_AODV has significantly low average packets in all levels of node mobility than both protocols as shown in Fig 6. In addition, with CP_AODV the number of RERR packets is reduced by more than $30 \%$ in average than the two protocols. Notice that both curves for RREP (Fig 6) and for RERR are similar to it for RREQ in Fig 4.

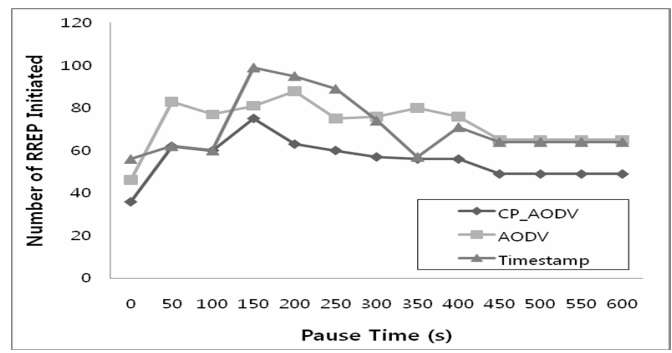

Fig. 6. The number of RREP packets initiated by a destination node

The proposed approach can save the amount of energy consumption by reducing the number of control packets including RREQs, RREPs and RERRs without increase in route discovery latency. The amount of energy consumption by CP_AODV, AODV and T-AODV are plotted against the pause time (i.e., node mobility) in Fig 7. Notice that the amount of energy consumption in Fig 7 includes both one for the route discovery process and one for data packet transmissions. Thus the shapes of the curves in Fig 7 are quite different from it in Fig 4. In all levels of node mobility, CP_AODV is remarkably less than AODV.

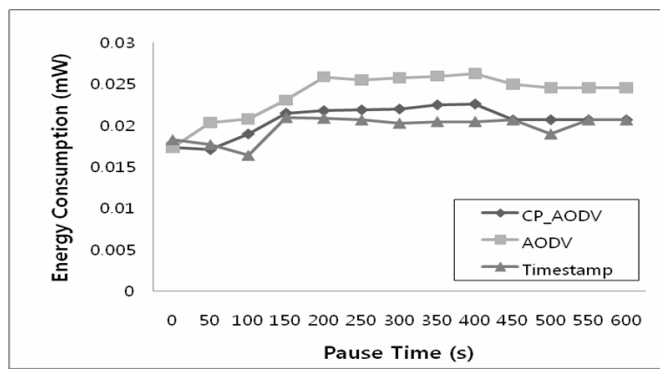

Fig. 7. Energy consumption versus node mobility

Notice that the amount of energy consumption is greater than T-AODV by $6 \%$ in average. The reason may be attributed to the fact that with T-AODV the number of data packets received by the destination node is relatively small. To be more precise, 
few observations can be made from our simulation results (Fig 8 and Fig 9). We see that there is no clear distinction for the throughput versus the pause time in Fig 8 for both CP_AODV and T-AODV. For the number of data packets received by the destination node, CP_AODV, however, is significantly higher than T-AODV by $10 \%$ as shown in Fig 8. We can say that T-AODV transmits less data packets compared to CP_AODV and thus its energy consumption becomes low.

CP_AODV achieves 5\% and 3\% better results for the throughput and the number of data packets received, respectively, compared to AODV irrespective of node mobility. From these observations, we can say that substantial reduction in the flooding of the control packets may result in a less route discovery latency. In turn, it causes low data packet traffic to increase the overall throughput.

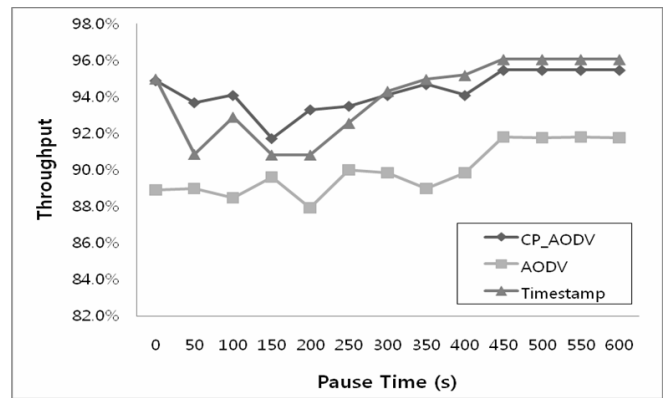

Fig. 8. The throughput versus the pause time

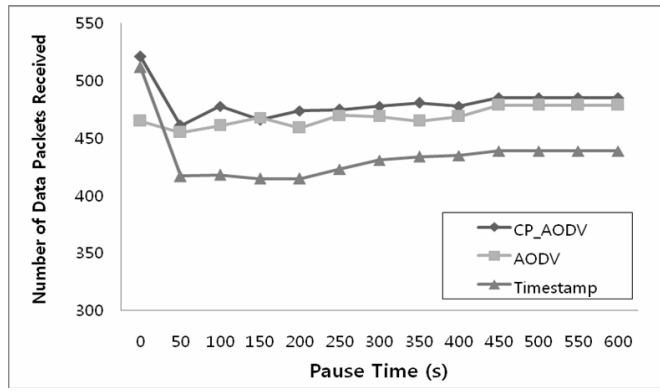

Fig. 9. The number of data packets received

\subsection{The Experiments for Node Velocity}

In this experiment each node moves with a nearly constant speed. When each node is set to the speed of $5 \mathrm{~m} / \mathrm{s}$, it moves with the speed of $4 \sim 5 \mathrm{~m} / \mathrm{s}$. At the experiments in the previous section, it moves with the random speed of $0 \sim 10 \mathrm{~m} / \mathrm{s}$. Thus, this section focuses on the performance evaluation under the node speed ranges. Through this experiment, the pause time is set to 100 seconds and thus the network has a high mobility. The other parameters are used in Table 3. As you see in Fig 10, the average number of RREQ packets of CP_AODV turns out to be better. More specifically, it of 
CP_ADOV has been measured 58.7, whereas that of AODV and that of T-AODV is 67.9 and 66.1, respectively. Besides, the standard deviation for the number of RREQs of $\mathrm{CP}_{-} \mathrm{ADOV}$ is the lowest among the three protocols. Thus, we can say that our proposed approach is more stable under the node speed ranges.

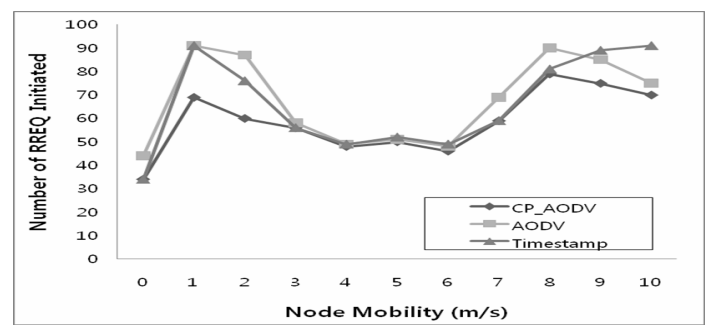

Fig. 10. The number of RREQ initiated versus node velocity

CP_AODV achieves substantial energy savings by $11 \sim 1.7 \%$ in average compared to both AODV and T-AODV. In addition, for the number of data packets delivered to the destination it is better than the two protocols by $14 \sim 2.4 \%$ in average (Fig 11). It cannot say that a network with slower movement of nodes achieves a better throughput than one with faster movement of nodes. In other words, to reduce the volume of control packets that needs to be exchanged between the source and the destination, all of the neighboring nodes keep the distance to exchange messages each other irrespective of node velocity.

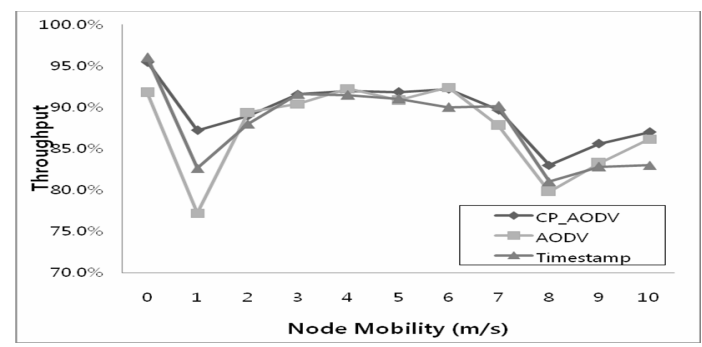

Fig. 11. The throughput versus node velocity

\subsection{The Experiments for Node Density}

In this experiment we vary the number of nodes starting from 36 up to 121 to evaluate the number of RREQ initiated (Fig 12), the amount of energy consumption and the number of data packets received by the destination (Fig 13). Since the rectangular field configuration is fixed as $1,500 \mathrm{~m} \times 1,500 \mathrm{~m}$, the distance between each pair of the neighboring nodes becomes shorter with a denser network. Depending on the number of nodes present in the ad-hoc network, we refer to the network as sparse which consists of less than 50 nodes. A dense network consists of more than 100 nodes. 
Through this experiment, the network has a high mobility. In the following results (Fig 12-13), we omit the results of T-AODV because they are similar to them in the previous sections. Thus, through this subsection, CP_AODV is directly compared to the legacy AODV for simplicity and clarity.

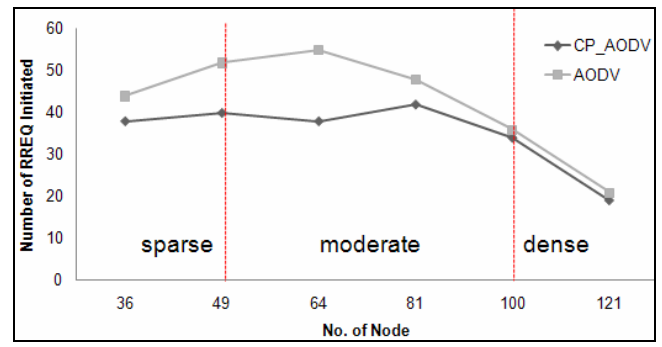

Fig. 12. The number of RREQ initiated versus the number of nodes

As in a dense network the number of pairs of the two neighboring nodes which locate within a possible transmission range of each other increase, the number of RREQ packets initiated by the source decreases significantly (Fig 12). That means in a dense network the possibility for finding a neighboring node to forward the request packets to the destination node is high. It causes the rate of RREQ packets initiated and forwarded to reduce remarkably. In a dense network, $\mathrm{CP}$ AODV performs similar to AODV as shown in Fig 13. In addition, in a dense network no clear difference between them occurs for the amount of energy consumption.

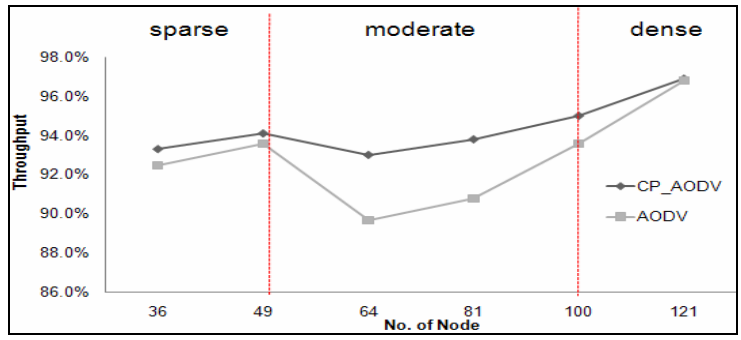

Fig. 13. The throughput versus the number of nodes

For sparse and moderate networks, overall performance of CP_AODV is found to be better than AODV. For the number of RREQ packets initiated, CP_AODV is 35.2 in average, whereas AODV is 42.7 in average. For both the amount of energy consumption and the number of data packets (Fig 13) CP_AODV is better than AODV by $13 \%$ and $2 \%$ in average, respectively. However, as the node density increases such the differences between them decreases. Thus our proposed method can achieve a better result with a sparse and moderate network than legacy AODV. 


\section{Concluding Remarks}

We proposed a control packet minimized AODV protocol for rapidly changing the network topology in ad-hoc wireless networks due to node mobility. During the route discovery process, the volume of the control packets increases with the changes in the network topology due to the movement of nodes. To reduce them significantly, more realistic estimation of NTT is needed. To minimize the flooding of control packets, a NTT should be adjusted smoothly based on AIAD technique. CP_AODV protocol provides good improvement in terms of the number of control packets, the amount of energy consumption, the throughput and the number of data packets received by the destination, compared to the legacy AODV and the modified AODV with timestamp technique. These results have been obtained under three distinct metrics; node mobility, node velocity and node density. However, in a dense network, CP_AODV performs similar to AODV, whereas it achieves a better result with a sparse and moderate network.

\section{References}

1. Corson, S., Macker, J.: Mobile Ad hoc Networking (MANET): Routing Protocol Performance Issues and Evaluation Considerations. RFC 2501 (1999)

2. Royer, E.M., Toh, C.-K.: A review of current routing protocols for ad-hoc mobile wireless networks. IEEE Personal Communications Magazine, 46-55 (1999)

3. Toh, C.K.: Ad-hoc Mobile Wireless Networks: Protocols and Systems. In: ACM SIGCOMM. Prentice Hall PTR (2002)

4. Perkins, C.: Performance Comparison of Two On-Demand Routing Protocols for Ad-hoc Networks. In: Proc. of the IEEE Infocom, pp. 3-12 (2000)

5. Ad hoc On-Demand Distance Vector (AODV) Routing (RFC 3561), http: / / www. faqs .org/rfcs/rfc3561.html

6. Carlberg, K., Crowcroft, J.: Building Shared Trees Using a One-to-Many Joining Mechanism. ACM Computer Communication Review 27(1), 5-11 (1997)

7. Hassan, J., Jha, S.: Optimizing expanding ring search for multi-hop wireless networks. In: GLOBECOM, pp. 1061-1065 (2004)

8. Tripathi, S.M., Ghosh, R.K.: An Implementational Study of Certain Heuristics for the Performance Enhancements of AODV. In: Proc. of Euro-Micro Workshop on Parallel, Distributed and Network-based Processing, pp. 395-402 (2002)

9. Lee, J., Park, H., Lee, K., Suk, K.: Performance Improvement of AODV Routing Algorithm Using Timestamp. Journal of Korea Information and Communication Society (domestic) 31(8), 830-836 (2006)

10. Kim, H.-c., Chunng, S.-m.: A study of Optimization using state space survey in ad hoc network. Journal of Korea Institute of Military Science and Technology (domestic) 8(4), 68$76(2005)$

11. Park, I., Pu, I.: Energy Efficient Expanding Ring Search. In: 1st ASIA Int. Conf. on Modeling \& Simulation (AMS), pp. 198-199 (2007)

12. http://www.scalable-networks.com 